\title{
Comparação do Peso dos Quelípodos e crescimento em duas Espécies de "Sirís" do Gênero Callinectes (Brachyura, Portunidae)
}

\author{
Cecilia Margarita Guerrero-Ocampo*, Maria Lucia Negreiros-Fransozo, Tânia Marcia Costa \\ NEBECC, Departamento de Zoologia -IB-e Centro de Aqüicultura - UNESP, “Campus” de Botucatu - Caixa \\ Postal, 510 - CEP 18600-000 Botucatu, SP, Brasil
}

\begin{abstract}
A comparison of the cheliped's weight in two species of the genus Callinectes was accomplished. The species $C$. danae e C. ornatus were collected by two otter trawl in Ubatuba bay $\left(23^{\circ} 26^{\prime} S\right.$ and $\left.45^{\circ} 02^{\prime} \mathrm{W}\right)$. The allometric constants obtained from the regression adjusted to a power function $\left(\boldsymbol{Y}=\boldsymbol{a} \boldsymbol{X}^{\boldsymbol{b}}\right)$ were analyzed. These species presented different allometry degrees for each sex considered. The relation $P Q \times P C$ presented positive allometry for sex of both species, but male presented higher positive allometry than female. $C$. danae presented higher positive allometry for chelipeds than $C$. ornatus. We suggest here that $C$. danae could be indicated to be submitted to grow out in ponds since it reaches higher size and bigger chelipeds.
\end{abstract}

Key words: Portunidae, Callinectes, siris, quelípodo, crescimento relativo, swimming crab, cheliped, relative growth.

\section{INTRODUÇÃO}

Os Portunidae representam $83 \%$ dos braquiúros coletados em fundos não consolidados da região de Ubatuba (SP). As espécies Callinectes ornatus e Callinectes danae destacam-se ocupando a primeira e a quinta colocação em abundância, com 67,96\% e 3,13\%, respectivamente (Fransozo et al., 1992). Este gênero tem se destacado por ter representantes de importância ecológica e pesqueira (PereiraBarros \& Travassos, 1975; Perry \& Malone, 1985; Oesterling \& Provenzano Jr., 1985; Pita et al., 1985).

Entre as relações biométricas, o estudo do crescimento relativo tem fornecido dados importantes para a carcinicultura. Este consiste na relação entre duas variáveis morfométricas, mostrando que o crescimento relativo de uma parte do corpo ou de um órgão em relação ao todo, pode ser descrita por uma equação matemática, sendo a do tipo potência, a mais utilizada (Rodrigues, 1985).
O conhecimento dessas relações tornam-se importantes à medida que tais informações possam auxiliar na seleção de espécies adequadas à comercialização ou, até mesmo, interessantes para o cultivo.

O quelípodo é uma estrutura notável do corpo dos braquiúros. Em caranguejos e outros decápodos, como nas lagostas, o quelípodo freqüentemente representa uma alta porcentagem do peso total do corpo (Stein, 1986) recebendo maior preço de comercialização no mercado.

A dimensão dos quelípodos de Callinectes danae e Callinectes ornatus foi estudada com o objetivo de avaliar o potencial destas espécies para futuros estudos sobre engorda em tanques de cultivo.

\section{MATERIAL E MÉTODOS}

Os "siris" foram coletados na Enseada de Ubatuba (SP) $\left(23^{\circ} 26^{\prime} \mathrm{S}\right.$ e $\left.45^{\circ} 02^{\prime} \mathrm{W}\right)$, com

\footnotetext{
* Author for correspondence
} 
barco de pesca de camarão equipado com duas redes de arrasto do tipo "otter trawl" com 7,5 m de comprimento e abertura de $15 \mathrm{~mm}$ entre-nós na panagem e $10 \mathrm{~mm}$ no saco.

As coletas consistiram de dois ou mais arrastos que foram realizados bimestralmente por um período de dois anos e meio (janeiro de 1991 a maio de 1993).

Após cada coleta, os exemplares foram identificados de acordo com Williams (1974), ensacados, etiquetados, congelados e transportados para o laboratório de carcinologia do Departamento de Zoologia -IB- UNESP, Botucatu.

No laboratório, as amostras foram descongeladas parceladamente à temperatura ambiente para o procedimento das análises.

Os dados registrados foram os seguintes:

a) sexo, baseado na forma do abdômen (em forma de " $T$ " invertido para machos; em forma triangular, para fêmeas jovens e, em forma de semicírculo, para fêmeas adultas);

b) peso úmido do corpo (PC) e peso do quelípodo (PQ) utilizando balança de precisão $(0,01 \mathrm{~g})$.

Os indivíduos com carapaças defeituosas ou regeneração incompleta dos quelípodos, as fêmeas ovígeras e animais em processo de muda recente, não foram utilizados.

Para a relação entre peso total $(\mathrm{P})$ e peso dos quelípodos (PQ), foi utilizada a função potência $\boldsymbol{Y}=\boldsymbol{a} \boldsymbol{X}^{\boldsymbol{b}}$, onde:

correspondente a PC; $\boldsymbol{X}=$ variável independente, a PQ;

$\boldsymbol{Y}=$ variável dependente, correspondente

$\boldsymbol{a}=$ coeficiente;

$\boldsymbol{b}=$ constante alométrica de crescimento.

Segundo Teissier (1960), a constante alométrica de crescimento $\boldsymbol{b}$ pode representar isometria $(b=$ 1), alometria positiva (b > 1) ou alometria negativa $(b<1)$. Por meio desta, pode-se inferir sobre as proporções em que as dimensões dos órgãos analisados crescem.
O ajuste da equação de regressão aos dados logaritmizados foi testado pela ANOVA da regressão. A alometria foi testada pelo teste " $t$ ". Os parâmetros $\boldsymbol{a}$ e $\boldsymbol{b}$ das equações para machos e fêmeas foram comparados usando o teste " $\mathrm{t}$ ". Todos os testes estatísticos foram realizados ao nível de significância de 5\% (Sokal \& Rholf, 1995).

\section{RESULTADOS}

Obteve-se um total de 2775 exemplares de $C$. ornatus e 2004 de C. danae, dos quais 599 exemplares de $C$. ornatus e 487 de $C$. danae foram utilizados nas regressões.

A distribuição de freqüência total por classe de tamanho (baseando-se na largura da carapaça, LC), para cada grupo de interesse de cada espécie é apresentada na Fig. 1. O maior número de indivíduos para machos de $C$. danae ocorre na classe de tamanho 78-] $86 \mathrm{~mm}$, enquanto que, para C. ornatus, encontrou-se na classe 61-] 69 $\mathrm{mm}$.

$\mathrm{O}$ peso médio dos quelípodos em indivíduos adultos difere entre as espécies estudadas, com $C$. danae apresentando quelípodos mais pesados que os de $C$. ornatus (Tabela I). O peso máximo dos quelípodos de indivíduos machos de $C$. ornatus foi de $22,50 \mathrm{~g}$ e de fêmeas $6,36 \mathrm{~g}$. Para os machos de $C$. danae o valor máximo obtido para os quelípodos foi de $35 \mathrm{~g}$ e para fêmeas, $12,20 \mathrm{~g}$.

Ao comparar-se as equações para machos e fêmeas das duas espécies (Tabela II), encontrou-se diferença quanto à constante de crescimento relativo $\boldsymbol{b}(\mathrm{p}<0,01)$, porém os interceptos $\boldsymbol{a}$ não foram significativamente diferentes $(p>0,05)$. 
Callinectes danae

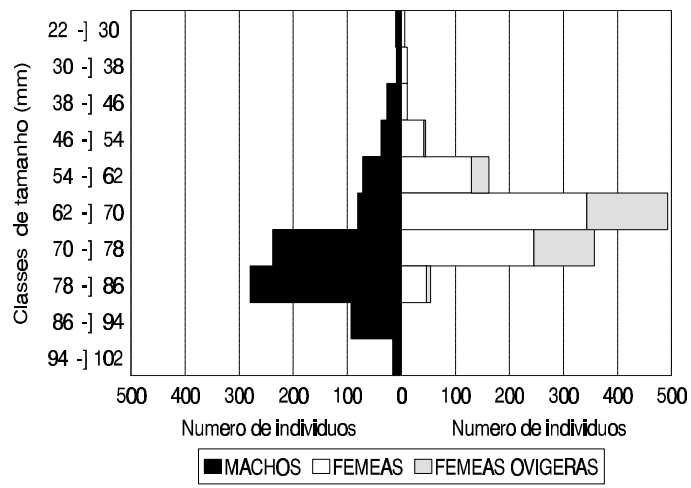

\section{Callinectes ornatus}

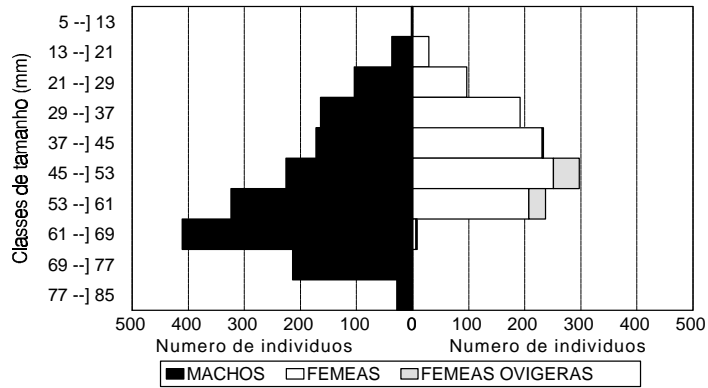

Fig. 1. Distribuição de frequiências por classes de tamanho de largura de carapaça $(\mathrm{mm})$ de Callinectes danae e Callinectes ornatus.

Tabela I. Peso médio dos quelípodos (media \pm desvio padrão) de adultos de cada espécie estudada

\begin{tabular}{lcc}
\hline Espécie & Macho & fêmea \\
\hline C. danae & $16,58 \pm 6,44$ & $6,88 \pm 2,33$ \\
C. ornatus & $9,59 \pm 3,86$ & $3,60 \pm 1,36$ \\
\hline
\end{tabular}

Tabela II. Equações alométricas para a relação: PQ x PC das espécies: Callinectes danae e Callinectes ornatus, com os respectivos coeficientes de determinação $\left(\mathrm{r}^{2}\right)$; $(\mathrm{MT}=$ machos totais, $\mathrm{FT}=$ fêmeas totais $)$.

\begin{tabular}{lccccccc}
\hline Espécie & Sexo & $\mathrm{n}$ & $Y=a X^{b}$ & $\mathrm{r}^{2(1)}$ & Alometria & $\mathrm{t}^{(2)}$ & $\mathrm{P}^{(3)}$ \\
\hline C. danae & & & & & & & \\
& $\mathrm{MT}$ & 285 & $\mathrm{PQ}=0,1023 \mathrm{PC}^{1,225}$ & 0,96 & + & 5,35 & $* *$ \\
& $\mathrm{FT}$ & 202 & $\mathrm{PQ}=0,1109 \mathrm{PC}^{1,140}$ & 0,97 & + & 10,38 & $* *$ \\
C. ornatus & & & & & & & \\
& $\mathrm{MT}$ & 373 & $\mathrm{PQ}=0,1418 \mathrm{PC}^{1,177}$ & 0,94 & + & 11,20 & $* *$ \\
& $\mathrm{FT}$ & 226 & $\mathrm{PQ}=0,1475 \mathrm{PC}^{1,100}$ & 0,95 & + & 5,86 & $* *$ \\
\hline
\end{tabular}

\footnotetext{
(1) da equação logaritmizada

(2) Teste $\mathrm{t}$

(3) $* *$ : $\mathrm{p}<0,01 ;$ n.s: $\mathrm{p}>0.05$
}

Na relação PQ x PC (Tabela II), a constante de crescimento relativo para cada espécie estudada indica uma alometria positiva, sugerindo que a taxa de ganho do peso relativo dos quelípodos é maior que a taxa de ganho de peso relativo do corpo. Evidenciou-se alometria positiva ligeiramente mais acentuada nos machos que nas fêmeas, de ambas espécies (Figs. 2 e 3).
As figuras 2 e 3 são resultado das funções potência plotadas entre os intervalos mínimo e máximo de PQ e PC de siris obtidos da natureza. Comparando-se as duas figuras, constata-se que, para $C$. danae, os machos alcançaram peso máximo de $125,0 \mathrm{~g}$, enquanto que as fêmeas alcançaram 64,0 g. Os valores máximos alcançados para $C$. ornatus foram menores, 78,4 g para machos e 27,6 g para fêmeas. 


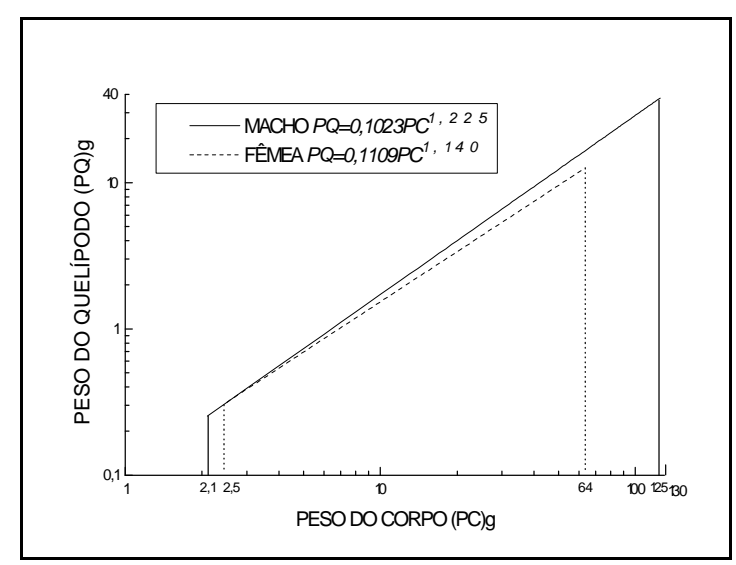

Fig. 2. Relação morfométrica entre peso do quelípodo (PQ) e peso do corpo (PC) de machos e fêmeas de Callinectes danae.

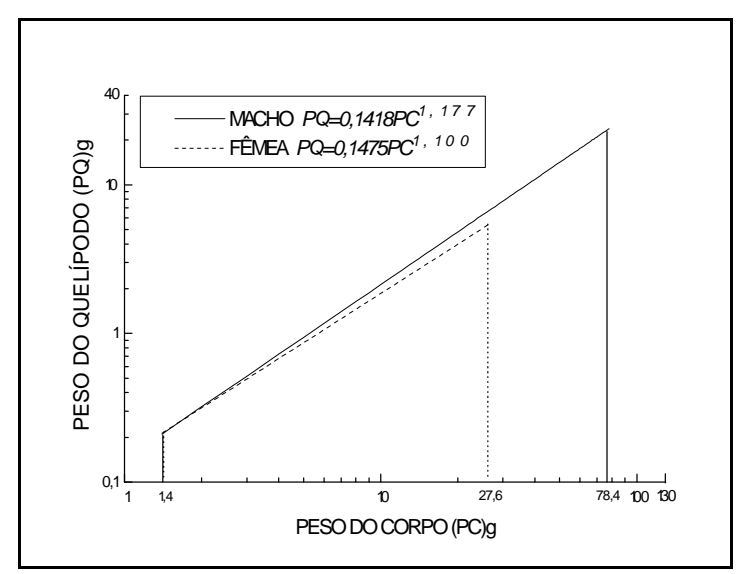

Fig. 3. Relação morfométrica entre peso do quelípodo (PQ) e peso do corpo (PC) de machos e fêmeas de Callinectes ornatus.

\section{DISCUSSÃO}

Segundo Vermeij (1977), na infra-ordem Brachyura, o dimorfismo sexual quanto ao tamanho das quelas revela que as fêmeas apresentam quelas menores que a dos machos da mesma espécie, sendo tal diferença mais acentuada em caranguejos rápidos e agressivos, como os gêneros Cancer, Menippe e Callinectes. Hartnoll (1974), notando tendências similares no dimorfismo sexual, sugere que a maioria de espécies nas quais os machos participam de interações agonísticas, o dimorfismo sexual é expresso no tamanho dos quelípodos, enquanto que, quando ambos sexos participam destes combates, tal diferença não é tão evidente.

Entre os portunídeos o caranguejo do mangue do Indo-Pacífico, Scylla serrata, apresenta as quelas maiores e mais robustas que as espécies do gênero Callinectes. As espécies do gênero Callinectes de águas tropicais do Atlântico são as que apresentam quelípodos maiores. No entanto exemplares de Callinectes sapidus de águas quentes não apresentam quelas significativamente maiores que as de águas temperadas (Vermeij, 1977).

Smith \& Palmer (1994) têm mostrado que o tipo de dieta também pode influenciar no tamanho da quela. Cancer productus criado experimentalmente com presas de conchas duras, desenvolvem quelas maiores e fortes do que aqueles alimentados com presas sem conchas, mas com nutrição equivalente.

O dimorfismo sexual detectado pela análise morfométrica dos quelípodos neste estudo, tem papel relevante no comportamento de acasalamento (exibição e corte) e para o sucesso reprodutivo, semelhante ao padrão descrito para outros braquiúros, como em Portunus pelagicus estudado por Fielder \& Eales (1972); Callinectes sapidus, por Churchill (1919) e Gleeson (1991); Hepatus pudibundus, por Reigada et al. (1994) e Arenaeus cribrarius, por Pinheiro (1995).

O acasalamento em Portunidae, ocorre tipicamente quando o exosqueleto da fêmea está mole. Durante esse período, que pode durar vários dias, antes e depois da ecdise (Hartnoll, 1969), a fêmea é protegida pelo macho. O tamanho maior da quela dos machos tem a função de auxiliá-lo na proteção da fêmea durante a muda puberal, quando a mesma está vulnerável a predadores. Zucker (1984) reporta que o tamanho médio da quela em machos de Uca musica terpsichores, que se encontrava em comportamento de corte, foi significativamente maior entre caranguejos de alta densidade populacional. Hartnoll (1974, 1978, 1982) documentou mudanças significativas em várias 
partes do corpo, incluindo a quela de caranguejos que atingiram a maturidade sexual. Tais mudanças, são claramente evidenciadas em machos de Carcinus maenas (Lee \& Seed, 1992) sugerindo uma provável função sexual.

Além do dimorfismo sexual, um polimorfismo sazonal também tem sido registrado em alguns decápodos (Hartnoll, 1982 Stein, 1986), onde os machos apresentam quelípodos maiores durante o período de atividade reprodutiva. As fêmeas de caranguejos, contrariamente, investem mais energia principalmente na produção de gametas, desde que a massa de ovos é da ordem de $10^{6}$ ovos (Warner, 1977). Durante o acasalamento, ocorrem conflitos entre machos, nos quais os quelípodos são os principais órgãos de agressão, sugerindo que o dimorfismo sexual no tamanho dos quelípodos seja resultado da seleção sexual.

Os machos atingem maiores pesos corporais e dos quelípodos que as fêmeas nas duas espécies aqui estudadas, sendo portanto, mais adequados para a engorda quanto a este aspecto.

No local de estudo, $C$. ornatus apresenta abundância de cerca de 23 vezes superior à de C. danae (Fransozo et al. , 1992), no entanto, sua importância para o cultivo é minimizada por não atingir um porte atrativo ao interesse comercial, como é o caso de $C$. danae.

O fato de $C$. danae atingir maior porte, quelípodos maiores, aliado à sua tolerância à variações de salinidade (Shumway, 1983), conferem forte justificativa para ser indicado para estudos de cultivo.

\section{AGRADECIMENTO}

À FAPESP pelo auxílio financeiro (Proc. $\mathrm{N}^{\circ}$ 91/2326-0 e 94/4878-9). Ao Dr. Wagner Cotroni Valenti e ao Dr. Marcelo A. Amaro Pinheiro, do Centro de Aqüicultura/UNESP, pelas valiosas sugestões e críticas ao manuscrito. À CAPES pela concessão da bolsa de mestrado para a primeira autora.

\section{RESUMO}

Neste trabalho, as espécies $C$. danae e $C$. ornatus foram comparadas quanto ao peso dos quelípodos. Os siris foram coletados com 2 redes de arrasto do tipo "otter trawl" na Enseada de Ubatuba $\left(23^{\circ} 26^{\prime} \mathrm{S}\right.$ e $\left.45^{\circ} 02^{\prime} \mathrm{W}\right)$. As constantes alométricas obtidas foram analisadas por meio da função potência $\left(\mathbf{Y}=\mathbf{a} \mathbf{X}^{\mathbf{b}}\right)$. As espécies apresentaram diferentes graus alométricos para cada sexo. A relação PQ X PC apresentou alometria positiva em ambas espécies, porém os machos apresentam maior alometria positiva do que as fêmeas. $C$. danae apresenta maior alometria positiva para os quelípodos do que $C$. ornatus. $\mathrm{O}$ fato de $C$. danae atingir maior porte e possuir quelípodos maiores indica que esta espécie tem potencial para o cultivo.

\section{REFERÊNCIAS BIBLIOGRÁFICAS}

Churchill JR., E. P. (1919), Life history of the blue crab. Bull. U.S. Bur. Fish., 36, 93-128.

Fielder, D. R. \& Eales, A. J. (1972), Observations on the courtship, mating and sexual maturity in Portunus pelagicus (L., 1766) (Crustacea: Decapoda: Portunidae). $J$. Nat. Hist., 6(3), 273- 277 .

Fransozo , A.; Negreiros-Fransozo, M. L.; Mantelatto, F. L. M.; Pinheiro, M. A. A. \& Santos, S. (1992), Composição e Distribuição dos Brachyura (Crustacea, Decapoda) do sublitoral não consolidado na Enseada da Fortaleza, Ubatuba (SP). Rev. Bras. Biol., 52(4), 667-675.

Gleeson, R. A. (1991), Intrinsic factors mediating pheromone communication in the blue crab, Callinectes sapidus, 17-32. In: Bauer, R. T.\& Martin, J. W. (Ed.) Crustacean Sexual Biology New York: Columbia University Press, 355p.

Hartnoll, R. A. (1974), Mating in the Brachyura. Crustaceana, 16, 161-181.

Hartnoll, R. A. (1974), Variation in growth pattern between some secondary sexual characters in crabs (Decapoda: Brachyura). Crustaceana, 27, 131-136. 
Hartnoll, R. A. (1978), The determination of relative growth in Crustacea. Crustaceana, 34, 281-293.

Hartnoll, R. A. (1982), Growth. In: Abele, L. G. (Ed.) The biology of Crustacea 2: Embryology, morphology, and genetics. New York: Academic Press, Pp. 111-196.

Lee, S. Y. \& Seed, R. (1992), Ecological implications of cheliped size in crabs: some data from Carcinus maenas and Liocarcinus holsatus. Mar. Ecol. Prog. Ser., 84, 151-160.

Oesterling, M. J. \& Provenzano Jr, A. J. (1985), Other crustacean species. In: Crustacean and Mollusk Aquaculture in the United States. New York: Van Nostrand Reinhold, Pp. 203234.

Pereira-Barros, J. B. \& Travassos, I. B. (1975), Informes sobre a pesca e a biologia do siri (Callinectes danae) e Guajaú (Callinectes bocourti), na Lagoa Mundaú-MaceióAlagoas. Recife: SUDENE-DRP, 9p. (Série Estudos de Pesca, 2).

Perry, H. M. \& Malone, R. F. (1985), National Symposium on the soft-shelled blue crab fishery. Missisipi: Gulf Coast Research Laboratory, $128 \mathrm{p}$.

Pinheiro, M. A. A. (1995), Biologia reprodutiva do siri chita Arenaeus cribrarius (LAMARCK, 1818) (Crustacea, Brachyura, Portunidae) na região de Ubatuba, litoral norte do estado de São Paulo. Tese (Doutorado)-Instituto de Biociências, Universidade Estadual Paulista. "Campus" Botucatu.

Pita, J. B.; Rodrigues, E. S.; Graça-Lopes, R. \& Coelho, J. A. P. (1985), Observações bioecológicas sobre o siri Callinectes danae, SMITH, 1869 (Crustacea, Portunidae), no complexo Baia-estuário de Santos, estado de São Paulo, Brasil. B. Inst. Pesca., 12(4): 35-43.

Reigada, A. L. D.; Negreiros-Fransozo, M. L. \& Mantelatto, F. L. M. (1994) Avaliação do Tamanho dos Quelípodos de Hepatus pudibundus (Herbst, 1785) (Crustacea, Calappidae), em relação ao sexo e à Maturação. Arq. Biol. Tecnol., 37(4), 797807, dez.
Rodrigues, S. A. (1985), Sobre o crescimento relativo de Callichirus major (Say, 1818) (Crustacea, Decapoda, Thalassinidae). Bol. Zool., 9, 135-211.

Shumway, S. E. (1983), Oxygen consumption and salinity tolerance in four Brazilian crabs. Crustaceana, 44(1), 76-82.

Smith, L. D. \& Palmer, A. R. (1994), Effects of manipulated diet on size and performance of Brachyura crab claws. Science, 264, 710712.

Sokal, R. R. \& Rholf, F. J. (1995), Biometry. New York: W. H. Freeman., 887p.

Stein, R. A. (1986), Sexual dimorphism in crayfish chelae: functional significance linked to reproductive activities. Can. J. Zool., 54, 220-227.

Teissier, G. (1960), Relative growth. In: WATERMANN, T. H. (Ed.) The physiology of Crustacea. New York: Academic Press, p. 537-560.

Vermeij, G. J. (1977), Patterns in crab claw size: the geography of crushing. Syst. Zool., 26, 138- 51.

Warner, G. F. (1977), The biology of crabs. London: Elek Science, 202p.

Williams, A. B. (1974), The swimming crabs of genus Callinectes (Decapoda, Portunidae). Fish. Bull., 72(3), 685-798.

Zucker, N. (1984), Delayed courtship in the fiddler crab Uca musica terpsichores. Anim. Behav., 32(3), 735-742. 